\title{
A NEW METHOD FOR CHARACTERIZATION OF CORONARY PLAQUE COMPOSITION VIA IVUS IMAGES
}

\author{
Arash Taki ${ }^{1}$, Alireza Roodaki ${ }^{2}$, Olivier Pauly ${ }^{1}$, S. K. Setarehdan ${ }^{3}$, Gozde Unal $^{4}$ and Nassir Navab ${ }^{1}$ \\ ${ }^{1}$ Department of Computer Aided Medical Procedures (CAMP), TU Munich, Munich, Germany \\ ${ }^{2}$ Department of Signal Processing and Electronic Systems, Supelec, Gif-sur-Yvette, France \\ ${ }^{3}$ Control and Intelligent Processing Center of Excellence, School of Electrical and Computer Engineering, College of Engineering, \\ University of Tehran, Tehran, Iran \\ ${ }^{4}$ Faculty of Engineering and Natural Sciences, Sabanci University, Turkey
}

\begin{abstract}
IVUS-derived virtual histology (VH) permits the assessment of atherosclerotic plaque morphology by using radiofrequency analysis of ultrasound signals. However, it requires the acquisition to be ECG-gated, which is a major limitation of VH. Indeed, its computation can only be performed once per cardiac cycle, which significantly decreases the longitudinal resolution of VH. To overcome this limitation, the introduction of an image-based plaque characterization is of great importance. Current IVUS image processing techniques do not allow adequate identification of the coronary artery plaques. This can be improved by defining appropriate features for the different kinds of plaques. In this paper, a novel feature extraction method based on Run-length algorithm is presented and used for improving the automated characterization of the plaques within the IVUS images. The proposed feature extraction method is applied to 200 IVUS images obtained from five patients. As a result an accuracy rate of $77 \%$ was achieved. Comparing this to the accuracy rates of $75 \%$ and $71 \%$ obtained using co-occurrence and local binary pattern methods respectively indicates the superior performance of the proposed feature extraction method.
\end{abstract}

Index Terms - IVUS, virtual histology, plaque characterization, texture analysis, support vector machine

\section{INTRODUCTION}

Acute coronary syndromes (ACS) and sudden cardiac death are the main causes of morbidity and mortality in the world [1]. They are mostly caused by the disruption of atherosclerotic plaque in an epicardial coronary artery.

The size and distribution of their main components, i.e. Dense Calcium (DC), fibrous, Necrotic Core (NC), and lipid rich tissues, permit to evaluate the risk of rupture [2].

Grayscale intravascular ultrasonography (IVUS) is a catheterbased technique, which provides the visualization of coronary atherosclerosis in vivo, elucidating lumen, vessel dimensions and the distribution of different plaques. Moreover, intra-luminal thrombus detection and plaque rupture assessment can be done by employing grayscale IVUS [3].

The tedious and time-consuming task of manual processing and interpretation of IVUS frames suffers from intra- and interobserver variability. Furthermore, an expert may not be able to completely characterize each type of atherosclerotic plaques. Thus automatic characterization of IVUS images is necessary.

The remaining of the paper is organized as follows: Section 2 describes the proposed method in detail. Section 3 provides the results obtained with the proposed method together with a
Virtual Histology ( $\mathrm{VH})$ is one of the well known techniques for analyzing IVUS data [4]. VH provides a color-coded plaque characterization through analysis and classification of the radiofrequency (RF) components of the backscattered ultrasound signal.

One of the major VH limitations is the ECG gated acquisition; i.e. only one frame is captured during a cardiac cycle. The end-diastolic point (peak R-wave) is chosen to acquire the desire frame as the heart is relatively at rest in this moment $[5,6$, 7, and 8]. Capturing only one frame per cardiac cycle with a constant pullback speed significantly decreases the longitudinal resolution of IVUS imaging.

To overcome this limitation, it is necessary to use all input IVUS images and characterize them with efficient textural features to permit the assignment of each pixel from the plaque area into one of the predefined tissue classes.

For this purpose, in [9] several feature extraction techniques are compared and they have reported that the co-occurrence method [10] has the best capability among their proposed methods. [11] Uses the gray-scale values by means of co-occurrence matrices in order to segment and classify regions of different plaques, with nearest neighbor learning technique. In [12], both signal and image based features are extracted. The co-occurrence, Local Binary Pattern (LBP) [13], and Gabor filtering methods are used for texture based feature extraction. Even though $90 \%$ accuracy is achieved, their approach is only validated with manually characterized IVUS images by two experts.

In this paper, we propose a novel feature extraction technique to provide a color-coded characterization of the atherosclerotic plaque directly from grayscale IVUS images. This can be performed on all the IVUS frames of the heart cycle and thus permits to outperform the longitudinal resolution of VH. It also gives the possibility of extracting additional information from the large archive of the IVUS images obtained in the past for which $\mathrm{RF}$ signals are not available. Our approach can be described as follows:

1) IVUS image is transformed into polar coordinates (2) Runlength matrices are computed from the plaque area of this polar image (3) Features are extracted from the run-length matrices (4) Each pixel is then classified into one of three tissue classes (i.e. DC, NC, and Fibro-lipid) with support vector machines. In vivo $\mathrm{VH}$ images, considered as gold standard for plaque assessment, are used for validation $[4,8]$.

comparison to those from co-occurrence and LBP feature extraction techniques. Finally section 4 concludes the paper. 


\section{METHODS}

In this paper, our task is to classify pixels from plaque area of IVUS images into three tissue classes. IVUS images are circular cross section of the blood vessel. Hence, input images are converted into polar coordinates to be orientation independent for the feature extraction. In the whole paper, $I$ always refers to the image in the polar domain.

\subsection{Run-length based textural descriptors}

Run-length transform [14] have been extensively used in segmentation and texture analysis. Let us consider the neighborhood centered on the pixel $(i, j)$ from image $I$. Its runlength matrix is defined in a certain direction as $R(a, b)$ where $a \in[1, P]$ is the gray level and $b$ the run-length, i.e. the number of consecutive pixels along a direction having the same gray level value. In this approach we characterize each neighborhood with two run-length matrices $R_{v}(a, b)$ and $R_{h}(a, b)$ corresponding respectively to vertical and horizontal directions.

Various textural features can then be derived from this runlength matrix. Such features have been previously used on IVUS images however the results were not fulfilling [9]. As shown in Fig. 1 (right column), most of the discriminative information lies in the homogeneity of specific intensities in different plaque types. Therefore, an efficient feature extraction method is one that is capable of encoding the homogeneity for each level of gray separately. Contrary to classical approaches where features are extracted from the whole run-length matrix, here we propose to extract two features characterizing each gray level $a$ (i.e. every row) of the run-length matrix. We denote them $f_{1}^{k}(a)$ and $f_{2}^{k}(a)$, where $k \in\{h, v\}$ refers to vertical or horizontal direction.

$f_{1}^{k}(a)$ is defined as the maximum number of occurrences multiplied by the length of the run with maximum occurrence $b_{m}$ :

$$
f_{1}^{k}(a)=R_{k}\left(a, b_{m}\right) \times b_{m}
$$

where $b_{m}=\underset{b}{\arg \max }\left(R_{k}(a, b)\right)$

$f_{2}^{k}(a)$ is defined as the sum of every occurrences multiplied by its corresponding run-length.:

$$
f_{2}^{k}(a)=\sum_{b} b \times R_{k}(a, b)
$$

Each pixel $(i, j)$ is characterized by a matrix $F_{i, j}$ of these descriptors, where $a$ refers to the grey level:

$$
F_{i, j}=\left(\begin{array}{cccc}
f_{1}^{v}(1) & f_{2}^{v}(1) & f_{1}^{h}(1) & f_{2}^{h}(1) \\
\vdots & \vdots & \vdots & \vdots \\
f_{1}^{v}(a) & f_{2}^{v}(a) & f_{1}^{h}(a) & f_{2}^{h}(a) \\
\vdots & \vdots & \vdots & \vdots \\
f_{1}^{v}(P) & f_{2}^{v}(P) & f_{1}^{h}(P) & f_{2}^{h}(P)
\end{array}\right)
$$

\subsection{Discriminative features extracted from spatial-frequency representation of descriptors}

Let us consider now each column $F_{i, j, c}$ of matrix $F_{i, j}$ as a signal function of the grey level $a$. As shown in Fig.1 (left column), these signals reveal different frequency contents. This motivates us to further characterize them by extracting discriminative features from its spatial-frequency representation. Therefore, each signal is decomposed in a detail $F_{i, j, c}^{1}$ and an approximation $F_{i, j, c}^{2}$ signal by using a 1D discrete wavelet transform (DWT):

$$
\begin{aligned}
& F_{i, j, c}^{1}[u]=\left(F_{i, j, c} * l\right)[u] \\
& F_{i, j, c}^{2}[u]=\left(F_{i, j, c} * h\right)[u]
\end{aligned}
$$

Where $h$ and $l$ are respectively the high and low pass filters used for the wavelet decomposition. The Daubechies 4 wavelet is used as mother wavelet for its ability to represent variations with a polynomial behavior of order 2 .

First, each DWT components (denoted by $F_{i, j, c}^{k}$ with $k \in\{1,2\})$ is characterized by a set of statistical features, namely its weighted mean, weighted variance, maximum of signal and its index:

$$
\begin{aligned}
& \rho_{1, c}^{k}=\frac{1}{P} \sum_{u=1}^{P} u \times F_{i, j, c}^{k}(u) \\
& \rho_{2, c}^{k}=\frac{1}{P} \sum_{u=1}^{P}\left(u \times F_{i, j, c}^{k}(u)-\rho_{1, c}^{k}\right)^{2} \\
& \rho_{3, c}^{k}=\max _{u}\left(F_{i, j, c}^{k}(u)\right) \\
& \rho_{4, c}^{k}=\underset{u}{\arg \max }\left(F_{i, j, c}^{k}(u)\right)
\end{aligned}
$$

Then, behaviors of these components have to be also characterized. By using an autoregressive (AR) model of order 5, the behavior of this signal can be predicted by:

$$
F_{i, j, c}^{k}(u)=\sum_{t=1}^{5} F_{i, j, c}^{k}(u-t) \times \varphi_{t, c}^{k}+C+\varepsilon_{u}
$$

where $C$ is a constant and $\varepsilon_{u}$ a noise. The coefficients of this AR model are used as further features:

$$
\rho_{t+4, c}^{k}=\varphi_{t, c} \quad \text { with } t \in\{1, \ldots, 5\}
$$

To summarize, each pixel $(i, j)$ is then described by a vector $X_{i, j}$ of features characterizing the spatial-frequency representation of textural information contained in its neighborhood:

$$
X_{i, j}=\left\{\rho_{t, c}^{k}\right\} \text { with } k \in\{1,2\}, t \in\{1, \ldots, 9\}, c \in\{1,4\}
$$

This vector is the input of the classifier described in the next section.

\subsection{SVM classifier}

In this paper the SVM is used as the classifier to find an optimal hyperplane separating samples from different classes by minimizing their misclassification error [15]. We consider the worst case of non-separable tissue classes. Thus, to increase their separability, a kernel function maps the input feature vectors to a higher dimension space. 


\section{RESULTS}

Our study group consists of sequences of IVUS images acquired from five patients. These images of size $400 * 400$ pixels were acquired using an electronic probe (In-Vision Gold, Volcano Therapeutics, Inc.) with a synthetic aperture $2.9 \mathrm{~F}$ and a frequency of $20 \mathrm{MHz}$. A motorized pullback was performed along the entire vessel with a speed of $1.0 \mathrm{~mm} / \mathrm{s}$ using a dedicated pullback device. Out of the frames in which all types of plaques were detected by the VH method, 40 frames were selected for each patient. The three mentioned methods were then applied on the set of 200 frames.

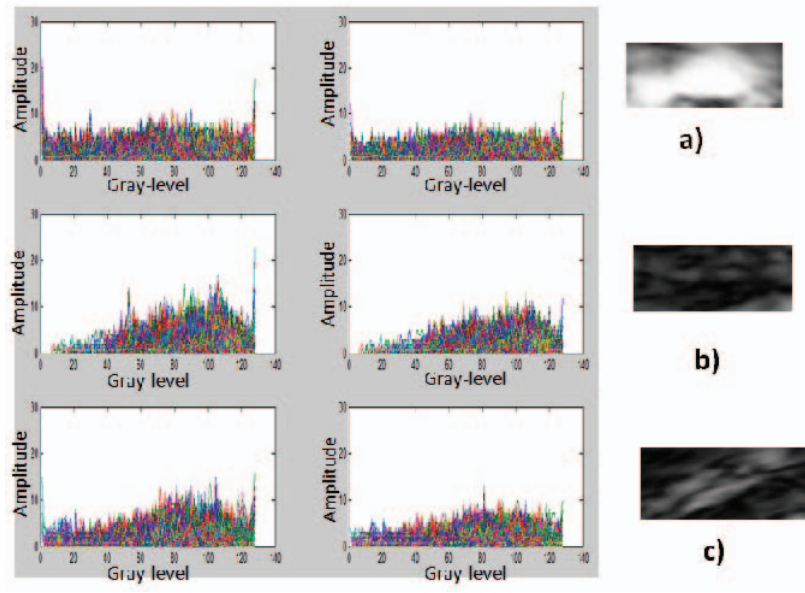

"Fig.1. Distribution of 5000 bundle feature vectors for each plaque type, (a) DC, (b) Fibro-lipid, and (c) NC. From left to right, the first and second column are illustrating the variation of the 1st feature (equation 1) and the second one (equation 2), respectively."

The characterized IVUS images were validated by their corresponding $\mathrm{VH}$ images and the accuracy, sensitivity, and specificity parameters were calculated for each technique. The results for our approach were compared to methods using LBP or co-occurrence-based features.

The size of the neighborhood was empirically chosen to be $11^{*} 11$ for all methods. This was done to get the optimum results for every method separately.

For LBP method, five circles were then constructed in each neighborhood. Then, three features were extracted from each circle ;the number of features for every pixel in LBP method thus sums up to 15 . Extracting features from five circles with different radii can be thought of as a multi-resolution textural analysis. Total number of features in the co-occurrence method was 14 which include e.g. homogeneity, contrast, inverse difference moment, and so on.
We used the publicly available $\mathrm{C}++$ implementation of the SVM algorithms known as LIBSVM [15]. A grid-search was performed for optimal parameter selection. A 5-fold cross validation to evaluate the performance of all three methods was applied. Results using different methods are presented below in Table 1. The proposed approach has more capability for classifying DC and NC plaques in comparison with LBP or cooccurrence methods.

Moreover, this approach reveals a higher overall accuracy. But co-occurrence and LBP methods performs better in characterizing the fibro-lipid regions. Fig.2 illustrates the images characterized by all methods with their corresponding IVUS and $\mathrm{VH}$ images.

In this study, the sensitivity of all methods for the detection of the NC was low (55\%). This fact was caused by similarities between NC and DC in Gray level IVUS images [16]. Furthermore, previous studies have showed that plaque areas adjacent to DC are frequently coded as $\mathrm{NC}$ tissue in $\mathrm{VH}$ images [17].

For a typical frame the proposed method took approximately 12 seconds to characterize the pixels, whereas the LBP took 2.6 minutes and the co-occurrence took nearly 60 minutes. Thus, in terms of time efficiency, the proposed method further outdoes the other two. A MATLAB implementation on an Intel Core 2 CPU $2.00 \mathrm{GHz}$ computer with $2.0 \mathrm{~GB}$ Ram was used in this work.

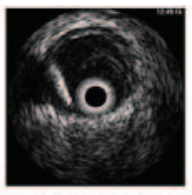

Sample IVUS image

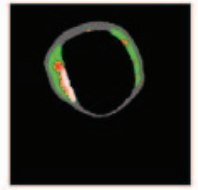

Proposed method

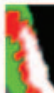

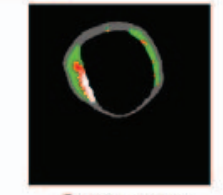

Co-occurrence

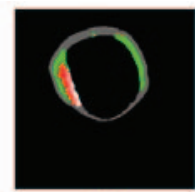

VH

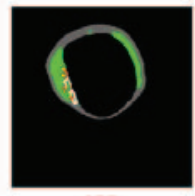

LBP
Proposedmethod Co-occurrence LBP

Fig. 2. The result of our feature extraction method in comparison with the Co-occurrence and LBP methods from left to right.

(White is DC, Green is fibro-lipid, and Red is NC)

Table I. The results of the proposed method versus other techniques

\begin{tabular}{|l|cccc|cc|c|} 
& \multicolumn{2}{|c}{ Dense Calcium (DC) } & \multicolumn{2}{c|}{ Fibro-lipid } & \multicolumn{2}{c|}{ Necrotic (NC) } & Overall \\
& Sensitivity & Specificity & Sensitivity & $\begin{array}{c}\text { Specificit } \\
\text { Accuracy }\end{array}$ & Sensitivity & $\begin{array}{c}\text { Specificit } \\
\text { A }\end{array}$ & \\
\hline LBP & $45 \%$ & $96 \%$ & $97 \%$ & $42 \%$ & $30 \%$ & $95 \%$ & $71 \%$ \\
\hline Co-occurrence & $67 \%$ & $95 \%$ & $84 \%$ & $80 \%$ & $53 \%$ & $83 \%$ & $75 \%$ \\
\hline Proposed method & $70 \%$ & $95 \%$ & $84 \%$ & $75 \%$ & $55 \%$ & $82 \%$ & $77 \%$ \\
\hline
\end{tabular}




\section{CONCLUSION}

In this paper, we presented a new image processing method for the characterization of arthrosclerosis plaque in IVUS images to improve the longitudinal resolution of $\mathrm{VH}$. Results showed the effectiveness of the proposed features in comparison to the cooccurrence and the LBP methods in terms of both time efficiency and classification accuracy. One of the limitations of the proposed features are their sensitivity to the artifacts especially the speckle noise since these artifacts tend to change the composition of the run length matrices by breaking potentially long runs into shorter runs, or by introducing runs with abnormal intensities.

In this study our IVUS image processing technique is evaluated against $\mathrm{VH}$, which is a signal processing approach. Since both of these techniques have their drawbacks, it would be of great interest to judge the proposed method against a ground truth obtained by histology.

As mentioned earlier, the number of classes was reduced to three classes instead of four, by combining the fibrous and fibrolipid classes. The next challenge is to further classify the fibrous and fibro-lipid areas.

\section{REFERENCES}

[1] E. J. Topol, TEXTBOOK OF CARDIOVASCULAR MEDICINE, Lippincott Williams \& Wilkins, 3rd ed., 2007.

[2] R. Virmani, F. D. Kolodgie, A. P. Burke, A. Farb, S. M. Schwartz, " Lessons From Sudden Coronary Death A Comprehensive Morphological Classification Scheme for Atherosclerotic Lesions," Arterioscler Thromb Vasc Biol., vol. 20, pp. 1262-1275, 2000.

[3] Di Mario CTS, Madretsma S, van Suylen RJ, et al, "Detection and characterization of vascular lesions by intravascular ultrasound: an in vitro study correlated with Histology". J Am Soc Echocardiogr, vol. 5, pp. 135-146, 1992.

[4] A. Nair, M. P. Margolis, B. D. Kuban, and D. G. Vince , "Automated Coronary Plaque Characterization with IVUS Backscatter_Ex Vivo Validation", EuroIntervention 2007.

[5]B. Lu, S.-S. Mao, N. Zhuang, et al, "Coronary artery motion during the cardiac cycle and optimal ECG triggering for coronary artery imaging," Invest Radiol, vol. 36, no. 5, pp. 250-256, 2001.

[6] A. U. Coskun, Y. Yeghiazarians, S. Kinlay, et al, "Reproducibility of coronary lumen, plaque, and vessel wall reconstruction and of endothelial shear stress measurements invivo in humans," Catheter Cardio Inte, vol. 60, no. 1, pp. 67-78, 2003.

[7] SA de Winter, R Hamers, et. al., "A Novel Retrospective Gating Method for Intracoronary Ultrasound Images based on Image Properties", Computers in Cardiology, vol. 30, pp. 13-16, 2003.

[8] K. Nasu, E. Tsuchikane, et. al., "Accuracy of In Vivo Coronary Plaque Morphology Assessment" Journal of the American College of Cardiology, Vol. 47, No. 12, 2006.
[9] D.G. Vince, K.J. Dixon, R.M. Cothren, J.F. Cornhill, "Comparison of texture analysis methods for the characterization of coronary plaques in intravascular ultrasound images", Computerized Medical Imaging and Graphics, vol. 24, pp. 221$229,2000$.

[10] Haralick R, Shanmugam K, Dinstein I. Textural features of image classification. IEEE Trans Syst Man Cyber, vol.6, pp: 610$621,1973$.

[11] X. Zhang, C. R. McKay, M. Sonka, "Tissue characterization in intravascular ultrasound images" IEEE Transaction on Medicine, Vol. 17, pp. 889-898, 1998

[12] S. Escalera, O. Pujol, J. Mauri, P. Radeva, "Intravascular Ultrasound Tissue Characterization with Sub-class ErrorCorrecting Output Codes", Journal of Signal Processing Systems,June2008.

[13] T. Ojala, M. PietikaÈ inen,, and T. MaÈenpa, "Multiresolution Gray-Scale and Rotation Invariant Texture Classification with Local Binary Patterns", IEEE Trans. PAMI, vol. 24, no. 7, 2002.

[14]S. Theodoridis and K. Koutroumbas, "Pattern recognition", Elsevier Academic Press, 5th ed., 2006.

[15] Chih-Chung Chang and Chih-Jen Lin, LIBSVM : a library for support vector machines, 2001. Software available at http://www.csie.ntu.edu.tw/ cjlin/libsvm

[16] N.Gonzalo, H.M Garcia, J. Lighthart et al,"Coronary plaque composition as assessed by grayscale intravascular ultrasound and radiofrequency spectral data analysis"J Cardiovasc Imaging, vol. 24, pp. 811-818, 2008.

[17] FJR Sales1, JLAA Falcão, et. al., "Evidences of Possible Necrotic-Core Artifact around Dense Calcium in Virtual Histology Images", Computers in Cardiology, vol. 35, pp. 545-548, 2008. 